\begin{tabular}{|rr|}
\hline Scientific & Research Article \\
& Sci. Res. Comm. (2022) \\
Volume 2, Issue 1 & Vosearch \\
\hline
\end{tabular}

\title{
Recovery of Pressed Titanium Alloy Machining Chip via Vacuum Induction Melting
}

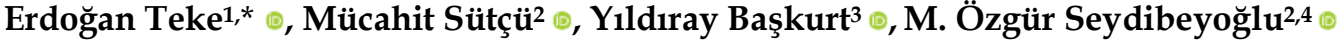 \\ ${ }^{1}$ Graduate School of Natural and Applied Science, İzmir Kâtip Çelebi University, Turkey \\ 2 Department of Materials Science and Engineering, İzmir Kâtip Çelebi University, Turkey \\ ${ }^{3}$ Department of Electrical and Electronics Engineering, Dokuz Eylül University, Turkey \\ ${ }^{4}$ Advanced Structures \& Composites Center, University of Maine, USA \\ *Corresponding author: erdoganteke@gmail.com
}

Received: 25.09.2021 Accepted: 27.01.2022

\begin{abstract}
Among many precious metals, titanium has been the first priority in various applications such as aerospace, automotive, marine and medical sectors due its superior mechanical, electrochemical, and biocompatible properties. In this study, a new melting method with vacuum induction system for Ti6Al4V alloy was developed to recover titanium scraps that were collected from medical implant machining industry. Prior to melting processing the recovered material was cleaned with ethyl alcohol and acetone ( $96 \%$ purity) to remove the coolant oil in the metal scraps. After the melting process, the microstructure, phase and hardness properties of melted bulk specimen were investigated by SEM, EDS, optical microscopy, XRD analysis and Rockwell hardness test. The results reveal that despite the high oxygen content, these molten bulk materials can be used in engineering applications other than biomaterials.
\end{abstract}

Keywords: Vacuum induction melting (VIM); titanium; waste scraps; recycling; microstructure.

\section{Introduction}

Titanium and its alloys are extensively used in many industries such as aerospace, biomedical, marine and automotive because of their good mechanical, physical and chemical properties (Froes, 2013), (Destefani, 1990), (Leyens \& Peters, 2003). The main advantages of titanium is its high strength-to-weight ratio and corrosion resistance. Its tensile and flexural strengths are comparable to steel, but the density is just the half of it (Leyens \& Peters, 2003), (Donachie, 2000). The most common used titanium alloy is Ti6Al4V, an $\alpha+\beta$ alloy, and has high toughness and working temperature, low density, excellent corrosion resistance and biological inertness it (Facchini et al., 2009), (Rack \& Qazi, 2006).

The most common manufacturing process of titanium-based products is usually computer numerical control (CNC) machining. In industrial production processes, the crucial waste titanium scraps are emerged after machining processes of its rods. The 
amount of scraps corresponds to $40 \%$ of the starting material used in the manufacturing of Ti6Al4V parts, and in some cases, much higher. These machining scraps are generally either collected in containers for recycling, or thrown into the waste storage area due to the risk of self-ignition. Disposed of Ti6Al4V alloy waste, which is in the category of precious materials, creates both environmental and economic problems ( Dikici \& Sutcu, 2017).

After machining, titanium scrap is generated in the forms of solid, turning and chip that result in the processing of bars, sheets, forge flashings, welding. The turnings usually contain some cutting lubricants; these wastes may also be contaminated with oil or grease (Neff, 1990). In recycling processes, the removal of these metal scrap from lubricant impurities is an important surface cleaning process. These pretreatments directly affect the quality of the materials to be produced in the recycling methods to be applied. Therefore, surface cleaning operations of scrap are important which can be costly and difficult.

In recent years, more than one manufacturing techniques such as melting and powder metallurgy processing for the recycling of precious metals such as titanium have been studied. In industrial applications, melting is generally a preferred method. Different melting methods such as vacuum arc re-melting (VAR) and electron beam melting (EBM) are available for the recovery of titanium scrap by melting. The melting of titanium alloys is carried out in common vacuum or an inert atmosphere vacuum (typically argon) (ASM Handbook, 2008). For the recycling of titanium and its alloys, it is important to note that titanium has a high affinity for oxygen and nitrogen (Topolski et al., 2017). Recently, some researches on the recycling of the titanium scraps with different methods were done by the researchers. Moon et al.(2017) focused on recycling of titanium scrap via the electromagnetic cold crucible (EMCC) technique (Moon et al., 2017). The scraps were successfully refined by this technique. Oh, \& Lim (2016) used vacuum arc-remelting (VAR) process to melt pure $\mathrm{Ti}$ and $\mathrm{Ti}$ alloys scraps in inert atmosphere $\left(\mathrm{Ar}-\mathrm{H}_{2}\right)(\mathrm{Oh}, \& \mathrm{Lim}, 2016)$. They investigated the effect of hydrogenplasma arc melting (HPAM) on the removal of metallic and gaseous impurities from pure titanium and titanium alloys. Su et al. (2009) studied the effect of hydrogen on the oxygen content of Ti6Al4V alloy through the remelting route in electric arc furnace (Su et al., 2009). Vutova et al. (2010) performed Ti from waste products by electron beam melting. The working vacuum pressure was $5-8 \times 10^{-3} \mathrm{~Pa}$ (Vutova et al., 2010). Roh et al. (2014) produced low oxygen content TiNi alloy powder from TiNi alloy scraps by using vacuum arc-remelting process (Roh et al., 2014). Ahmed et al. (2017) focused on the production of well-finished part made of Ti6Al4V from the powder by EBM (Ahmet et al., 2017).

Induction heating is the process of heating an electrically conducting part by passing an alternate current at a specific frequency through a copper coil to generate a variable electromagnetic field, which generates induced eddy currents in the vicinity of the coil. The eddy currents flowing through the part's resistance, which is placed within the coil, heat it via the Joule effect. These eddy currents are caused by the part's resistance and result in power loss. This power loss manifests itself as heat. The vacuum induction melting process exhibits several advantages: low cost, short melting time, homogeneous mixing, precise control of the temperature, easily accessible and being energy efficient (Sazak, 1999), (Rudnev et al., 2003).

In this study, a new melting system with vacuum induction for Ti6Al4V alloy was developed to recover the titanium chip that was collected from medical implant 
machining industry. After the vacuum induction melting process, the physical, microstructural and mechanical properties of the melted bulk Ti6Al4V sample was investigated.

\section{Materials and method}

\subsection{Preparation of Ti6Al4V alloy chip}

Ti6Al4V alloy scraps were used as the initial materials in this study in the chip form obtained by CNC machining. Chip cleaning is also one of the main factors that determines the quality of melt material. High level of contamination with foreign particles will affect the elemental composition. During machining, the surface of the titanium chip is contaminated with lubricating, cooling oil or oxide. Prior to melting processing, the titanium scraps were cleaned with detergent and ethyl alcohol $(96 \%$ purity) to remove the coolant oil. The Ti6Al4V scraps were crushed in chips form under $5 \mathrm{~mm}$ to reduce the size by mechanical crusher. Then, the reduced size chip was compressed into a cylindrical briquette by applying 170 tons pressing in a hydraulic press. The Ti6Al4V scraps in the form of chips and briquettes prepared before melting are given in Figure 1. The Ti6Al4V alloy has low thermal and electrical conductivity. In order to make a quick and easy melting process, the pores or voids inside the briquettes must be at a minimum level. For this reason, reducing the size of the chip facilitates both the pressing process and the formation of briquettes with less pores.
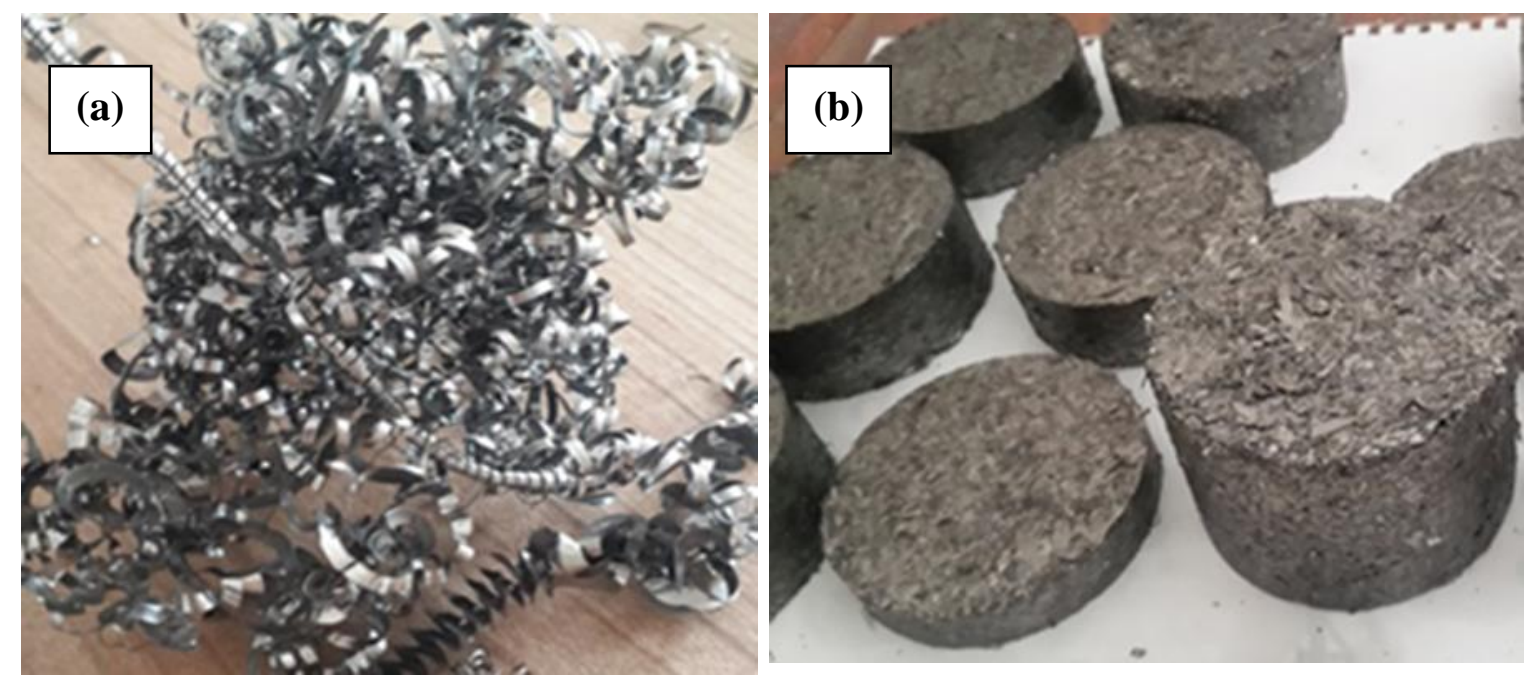

Figure 1. (a) The Ti6Al4V chip and (b) the briquettes pressed from their chip

\subsection{Vacuum induction melting (VIM) system}

The melting process was performed within a low pressure chamber equipped with a water-cooled induction coil, as it is given schematically in Figure 2. Here, the induction coil was operated with a medium frequency power supply. 


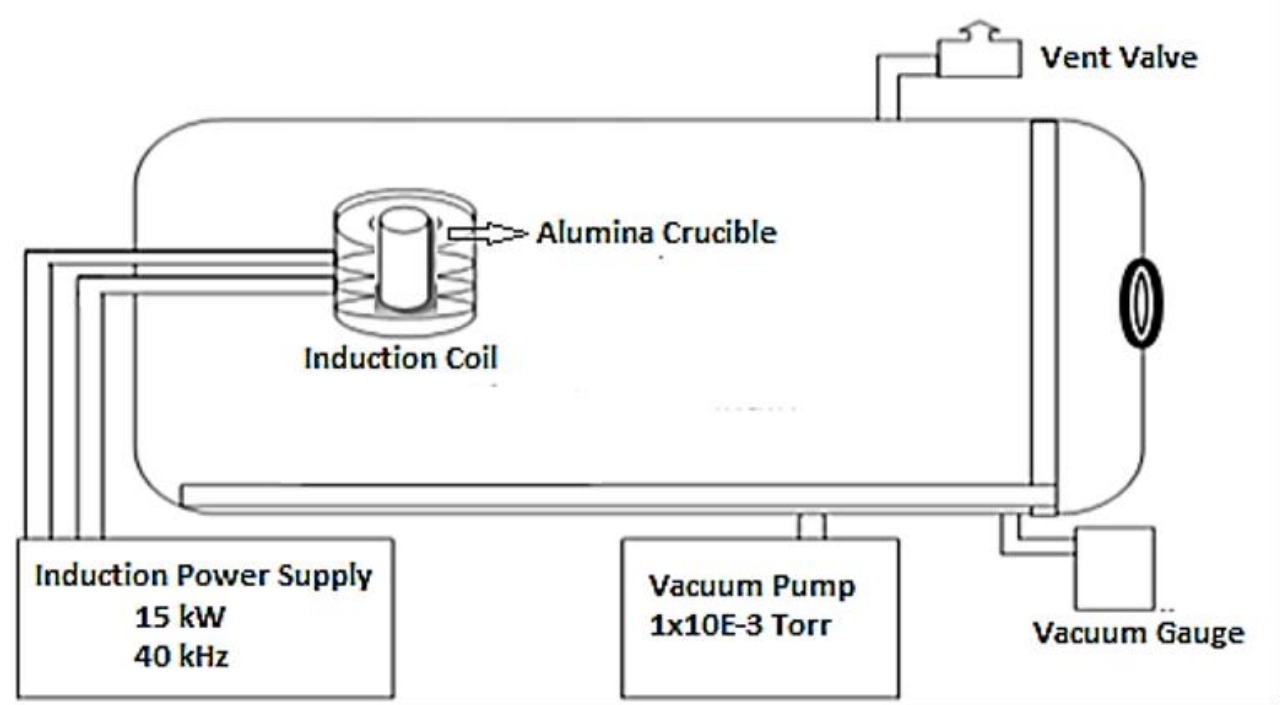

Figure 2. The vacuum induction melting (VIM) system

Prior to melting process, the base pressure of the system was maintained at $1 \times 10^{-3}$ torr for 30 minutes to minimize the presence of foreign particles in the environment. The vacuum value of the system, the applied power and the crucible material are important parameters affecting the mechanical and chemical properties of the obtained sample. The melting process was carried out at pressure of $2,1 \times 10^{-2}$ torr due to the resulting heat. Alumina crucible with $99,8 \%$ purity was used because of its resistance to oxidation at high temperatures. The induction power was applied gradually to prevent the crucible from being affected by thermal shocks. A medium frequency induction generator with a power of $15 \mathrm{~kW}$ was specially produced for the melting process. One of the most basic parameters of the generator design is to determine the operating frequency. Alternating currents can flow efficiently from the surface to a certain depth depending on their frequencies, the magnetic and electrical properties of the conductor through which they pass. However, the chip was pressed under high pressure and turned into briquettes. Thus, the pressed compacts are not in the form of a uniform structure and they don't have a uniform structure of completely discrete particles. Each chip particle touches one another mechanically but is not in absolute contact with possible oxidation and pollution causes. The application frequency with these assumptions was chosen empirically in the order of $40 \mathrm{kHz}$. During the melting process, the operating frequency was measured as $39.67 \mathrm{kHz}$. After melting, the specimen was allowed to cool in vacuum for 1 hour.

For microstructural investigation, the specimen was cut with diamond disc and then its surface were grinded with silicon carbide (SiC) grinding papers of 180-320-600-1200 grit, respectively. Then, the VIM molten Ti6Al4V sample surfaces were polished with diamond solutions of 9-3-1 $\mu \mathrm{m}$, respectively. To reveal the microstructure of polished surface, chemical etching on the sample surface was carried out using an etchant solution composed of $10 \mathrm{ml}$ of hydrofluoric acid $(\mathrm{HF}), 5 \mathrm{ml}$ nitric acid $\left(\mathrm{HNO}_{3}\right)$, and 85 $\mathrm{ml}$ of water.

\subsection{Characterization}

The phase analysis of the VIM molten Ti6Al4V sample was carried out using a Bruker Phaser D2 diffractometer with a Cu-Ka x-ray $(\lambda=1.5404 \AA ; 30 \mathrm{kV})$ diffraction beam. 
The elemental analysis and morphology of VIM molten Ti6Al4V sample and chip were investigated by using SEM-EDS, by Carl Zeiss 300VP scanning electron microscopy with Energy Dispersive Spectrometry (EDS). The crystalline phase formations and microstructure of the VIM molten Ti6Al4V were examined by optical microscope (OM) by using Nikon Eclipse LV150N. HRC hardness tests preloading at $98 \mathrm{~N}$ and max. loading at $1471 \mathrm{~N}$ in 3 seconds were conducted at room temperature using a Prüfen+Messen Tester machine. The density of the VIM molten Ti6Al4V sample was determined using Archimedean method at room temperature. The surface roughness of the VIM molten Ti6A14V sample was obtained with a Mitutoyo SJ-210 profilometer.

\section{Results and Discussion}

\subsection{XRD analysis}

X-ray diffraction spectra of commercial bar, chip and VIM molten Ti6Al4V is given in Figure 3 and Figure 4, respectively. The patterns presented for each sample are mostly consist from a (hexagonal close packed-hcp) phase.

XRD patterns of the chip and VIM molten Ti6Al4V show similar diffraction patterns. $a$ and $\beta$ (body centered cubic-bcc) phase peaks are different only as to intensity. The peak intensities of VIM molten Ti6Al4V are almost the same as commercial Ti6Al4V bar but slightly higher than the Ti6Al4V chip spectrum because of the finer structure in VIM molten Ti6Al4V.

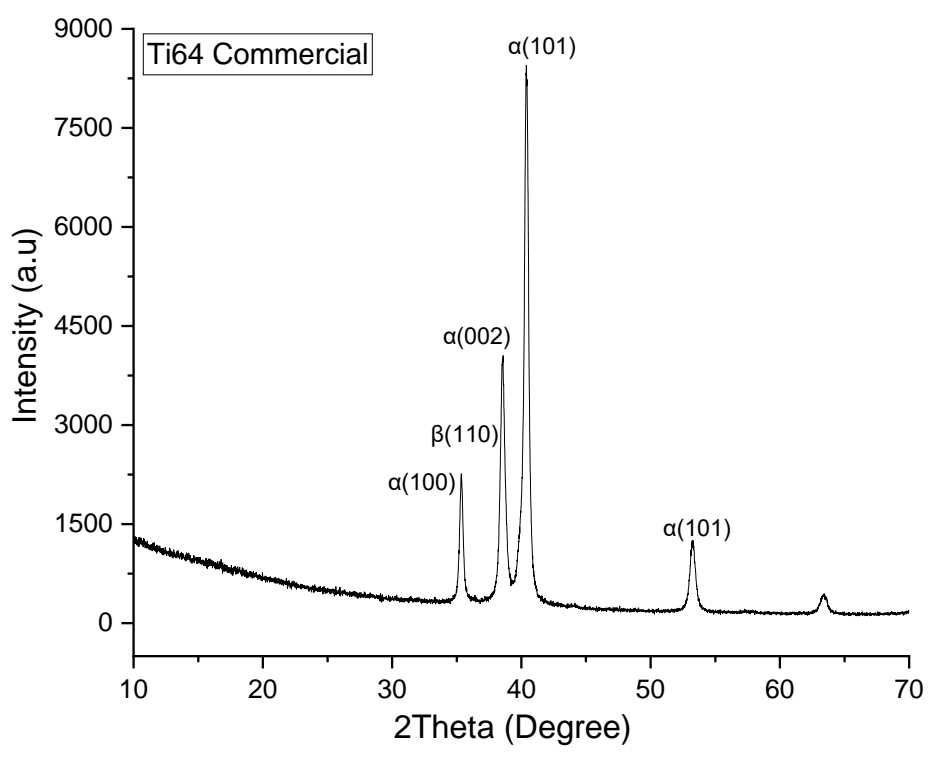

Figure 3. X-ray diffraction spectra of commercial Ti6Al4V bar

Between about $35^{\circ}$ and $41^{\circ}$, the peaks occured for the planes (110) given by the body centered cubic structure, and also by planes (100), (002) and (101) of hexagonal close packed structure. The low intensity (110) peak indicates that a small amount of $\beta$ phase is maintained in the final microstructure. Thereby, it is approved that the microstructure of VIM molten Ti6Al4V consists of $\alpha$ and $\beta$ phases. 


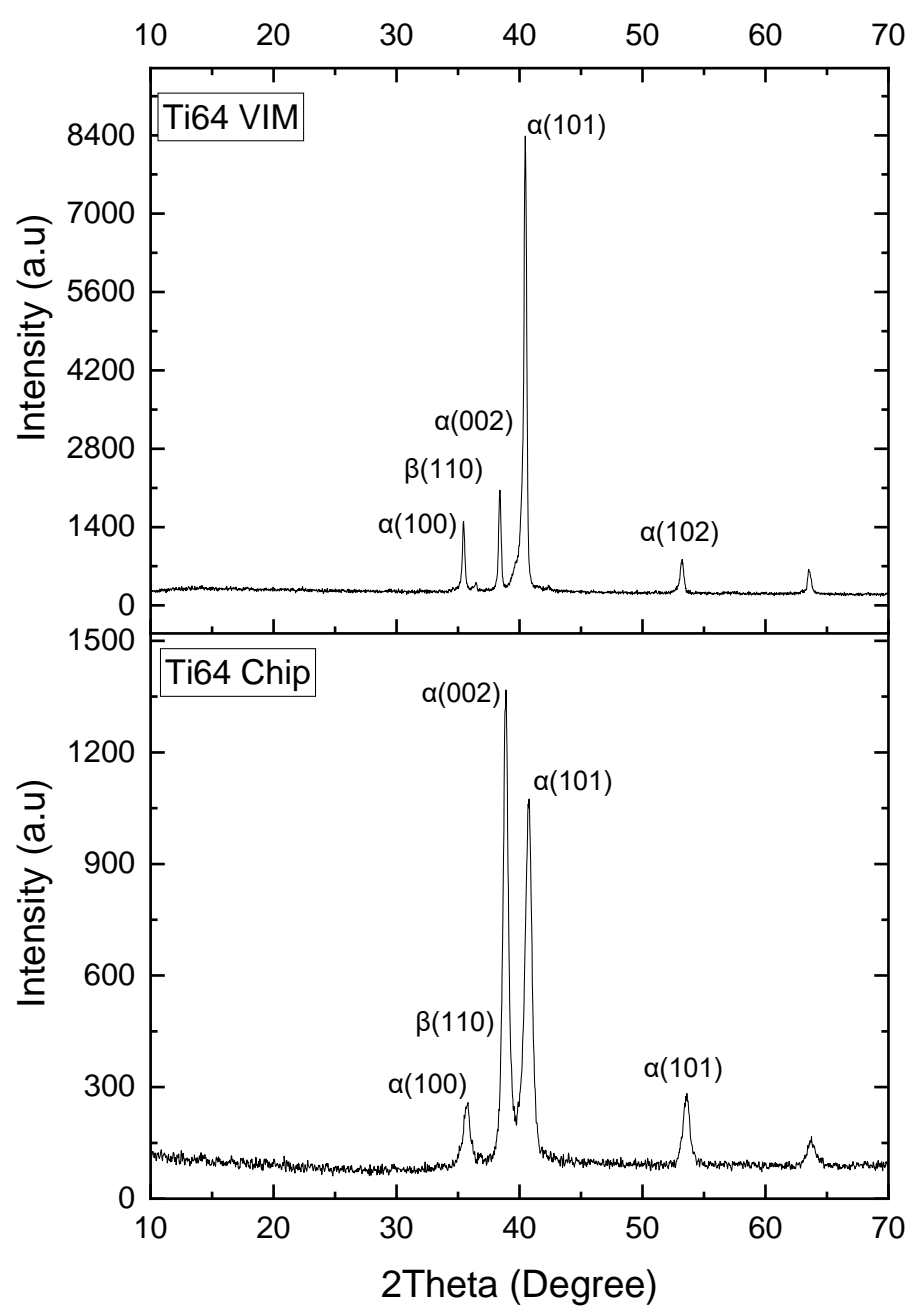

Figure 4. X-ray diffraction spectra of Ti6Al4V samples; chip and VIM molten

After melting, the crystallographic orientation of the VIM molten sample was reorganized and finally the crystal structure was formed that have the grains with (101) plane. The phase analysis results are consistent with the values presented in literature. According to Formanoir et al. (2016) and Balla et al. (2014) the final results support the microstructural changes related to phase transformation and indicate that VIM molten Ti6Al4V contain a (hcp) and $\beta$ (bcc) phases after melting process. In addition, compared to chip and VIM molten Ti6Al4V, the peak intensities of a phases increased considerably after melting.

The crystal size of the samples was calculated using the Debye-Scherrer equation which is Equation (1) (Suryanarayana, 2001):

$$
\mathrm{D}=\mathrm{K} \lambda / \mathrm{B} \cos \Theta
$$

where $\mathrm{K}(0,9)$ is the numerical factor often called the crystallite-shape factor, $\mathrm{D}$ is the crystallite size, $\lambda$ is the wavelength of the $\mathrm{X}$-rays, $\mathrm{B}$ is the peak full width at half of the maximum intensity (FWHM) in radians, and $\Theta$ is the Bragg angle in radians. The crystal size of the samples is given in Table 1. According to this, the average crystal size of the commercial Ti6Al4V bar, chip and molten sample was calculated as about 18.5, 
13.1 and $29.2 \mathrm{~nm}$, respectively. It was found that the crystal size of the molten sample was larger than the crystal size of the commercial Ti6Al4V bar and chip.

Table 1. The crystallite size of the Ti6Al4V samples

\begin{tabular}{ccccc}
\hline $\begin{array}{c}\text { Sample } \\
(\text { Ti-6Al-4V) }\end{array}$ & $\begin{array}{c}\text { Peak position } \\
(2 \Theta)\end{array}$ & $\begin{array}{c}\text { FWHM } \\
(\mathrm{B})\end{array}$ & $\begin{array}{c}\text { Crystallite size } \\
(\mathrm{D})(\mathrm{nm})\end{array}$ & $\begin{array}{c}\text { Average D } \\
(\mathrm{nm})\end{array}$ \\
\hline \multirow{4}{*}{ VIM molten } & 35.45 & 0.265 & 31.39 & \\
& 38.40 & 0.258 & 32.56 & 29.25 \\
& 40.46 & 0.315 & 26.86 & \\
& 53.20 & 0.350 & 25.37 & \\
Chip & 63.56 & 0.310 & 30.08 & 13.17 \\
& 35.71 & 0.674 & 12.36 & \\
& 38.88 & 0.565 & 14.88 & 18.51 \\
\hline \multirow{5}{*}{ Commercial } & 40.71 & 0.753 & 11.24 & \\
bar & 53.57 & 0.652 & 13.63 & \\
& 63.72 & 0.680 & 13.73 & \\
& 35.35 & 0.332 & 25.05 & \\
\hline
\end{tabular}

\subsection{Optical Microscopy analysis}

Figure 5, which clearly shows fine needle-shaped structure with multi-directional distribution, represents the optical micrographs of VIM molten Ti6Al4V sample. The microstructure of VIM molten sample essentially consists of an a phase and a small amount of $\beta$ within columnar grains.

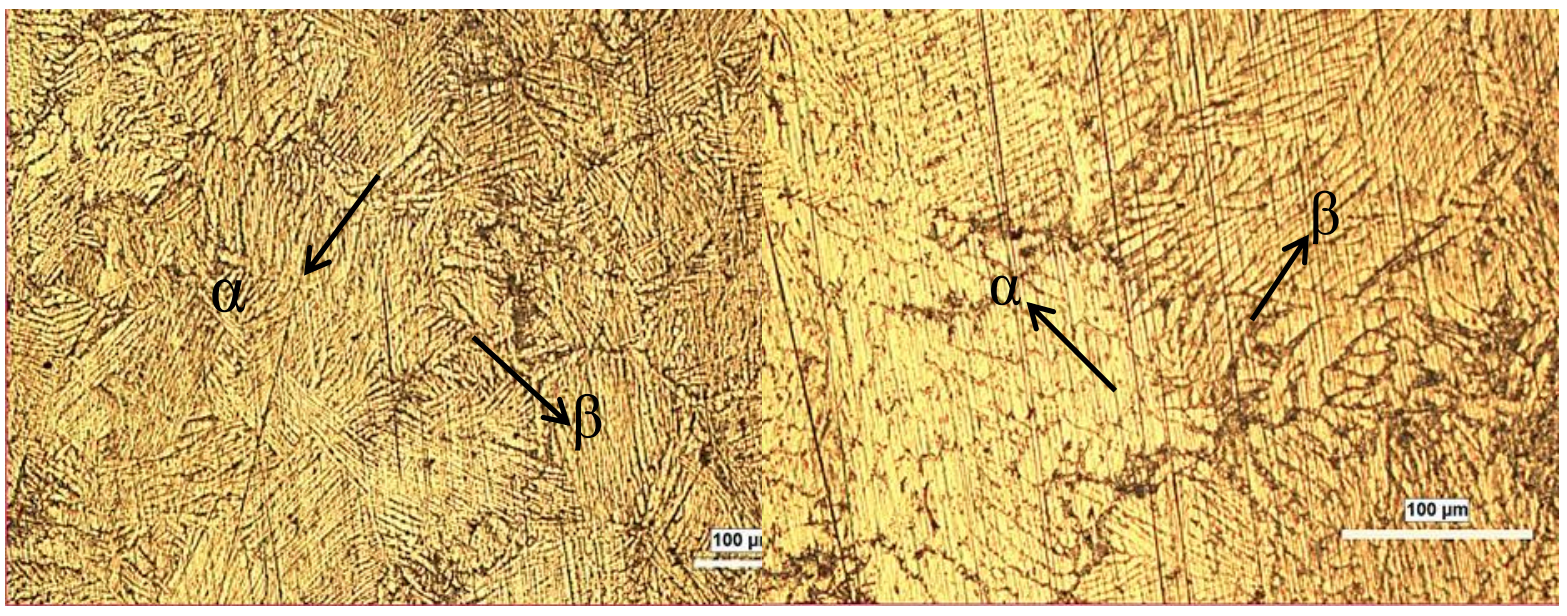

(a) $100 \mathrm{X}$

(b) $200 \mathrm{X}$

Figure 5. Optical micrographs at different magnifications of VIM molten Ti6Al4V 
The a phase possesses a lamellar morphology with $\beta$ surrounding the a lamellar boundary. However, $\beta$ columnar grain boundaries are obviously observed. $\alpha$ (hcp) phases are shown in bright surface and $\beta$ (bcc) phases are seen in regions indicated by dark surface. The lamellar of the a phase (shown light) are relatively regular. The microstructure of VIM molten sample is lamellar due to resulting from rapid cooling from the high-temperature $\beta$ phase. This was previously reported by Safdar et al. (2012) and Reginster et al. (2013).

A small pores were formed during melting as shown in Figure 6. The pores are mostly flat or irregular-shaped and formed at the interface between grain boundaries and the sizes of the pores range from $10 \mu \mathrm{m}$ to $30 \mu \mathrm{m}$ in diameter. The pores are caused by residual stresses due to increased temperature rapidly and rapid cooling afterwards and incomplete solidification (Qiu, \& Attallah, 2013), (Vilaro et al., 2012), (Amato et al., 2012), ( Thijs et al., 2010), (Facchini et al., 2010). The minimum porosity level that can be achieved in the molten sample is around $0.45 \%$. The density of the molten sample was calculated as $4.41 \mathrm{~g} / \mathrm{cm}^{3}$. It is very close to the density of the commercial Ti6Al4V alloy $\left(4.43 \mathrm{~g} / \mathrm{cm}^{3}\right)$.

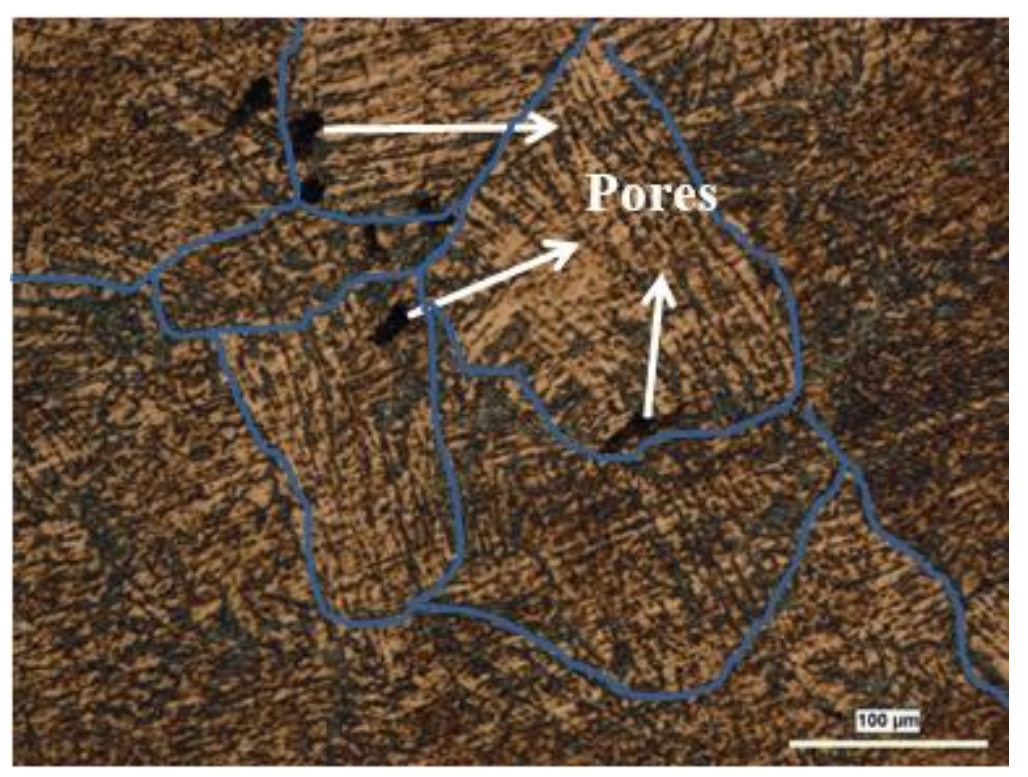

Figure 6. The pores in VIM molten Ti6Al4V sample

Moreover, surface roughness range of the VIM molten sample is very restricted after polished and the average roughness $\mathrm{Ra} 0.08 \mu \mathrm{m}$. The roughness measurement results show that a quite fine surface could be obtained by VIM.

\subsection{SEM and EDS analysis}

Figure 7 indicates SEM images taken from the rough and smooth sides of Ti6Al4V chip. During machining process of Ti6Al4V, the workpiece undergoes large deformation at high strain rate and temperature, which can change the microstructure and material properties of the $\mathrm{Ti}$ material and chip. Therefore, for surface characterization of Ti6Al4V chip, EDS was used to investigate the surface compositions after the cleaning process. 


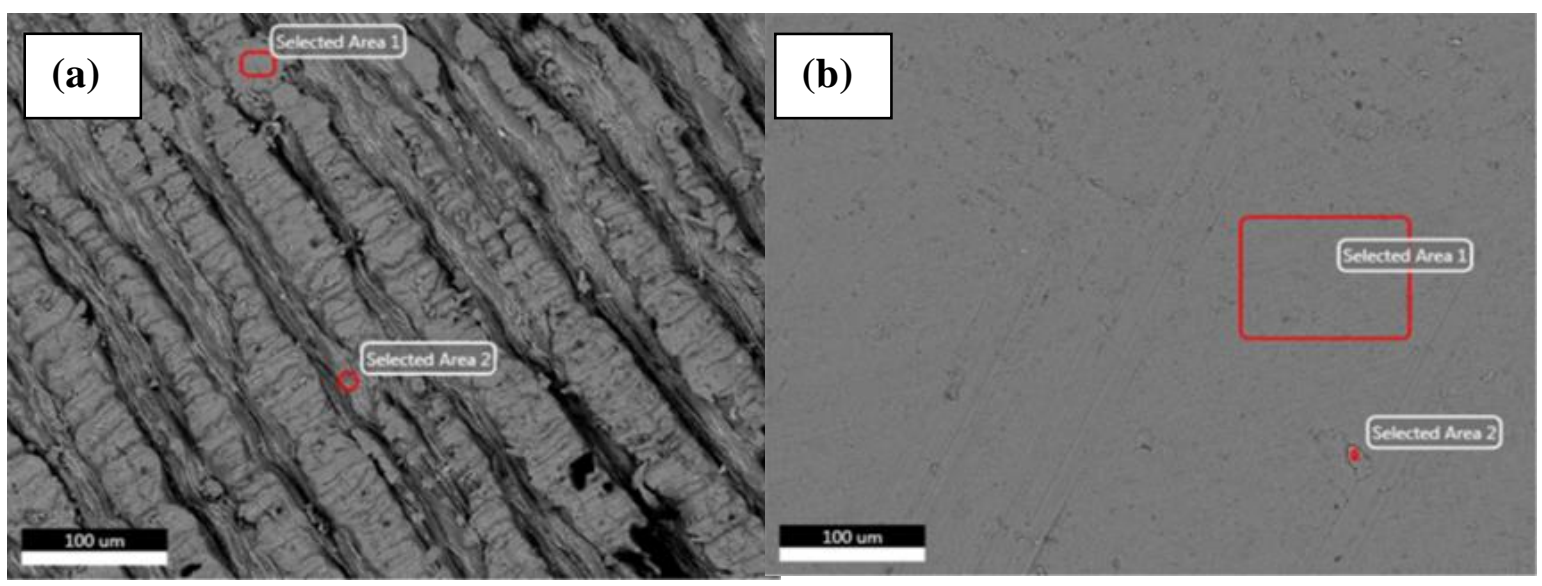

Figure 7. SEM images and EDS point analysis of Ti6Al4V chip at 500x magnification (a) rough side, $(b)$ smooth side

The elemental distribution of the selected regions on the chip is given in Table 2. EDS analysis of both side of chip showed that oxygen $(\mathrm{O})$ and carbon $(\mathrm{C})$ ratios are high.

Table 2. Elemental composition of the Ti6Al4V chip

\begin{tabular}{|c|c|c|c|c|c|}
\hline \multicolumn{1}{|c|}{} & \multicolumn{3}{c|}{} \\
\hline
\end{tabular}

The effect of machining atmosphere and temperature are considered as important reasons. Thus, the locate surface of impurity atoms such as $\mathrm{O}$ and $\mathrm{C}$ accelerate. Another factor can be interpreted as the fact that the waste chip can not be completely purified from the impurities as a result of the washing process. SEM images and selected areas for EDS analysis of molten Ti6A14V sample is given in Figure 8. 


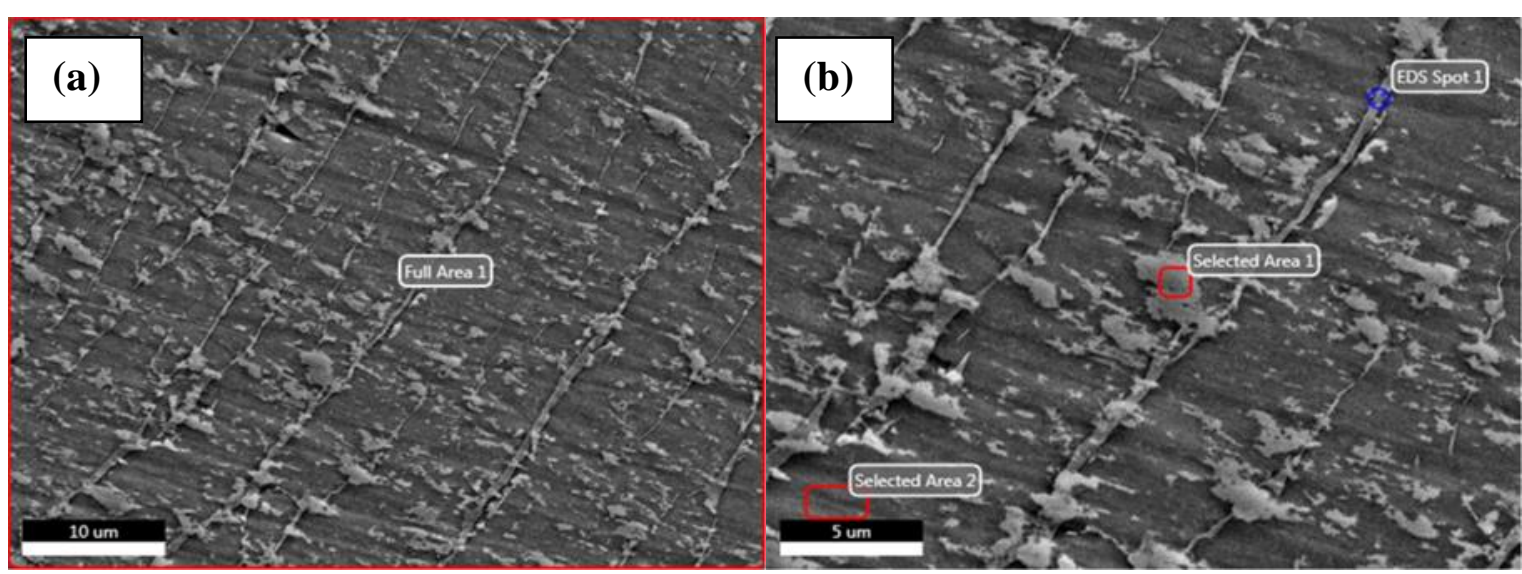

Figure 8. SEM images and EDS analysis of VIM molten Ti6Al4V sample at different magnifications of (a) 500X and (b) 2500X

The chemical composition of VIM molten Ti6Al4V was determined by EDS analysis, given in Table 3.

Table 3. Elemental distribution (EDS) of VIM molten Ti6Al4V sample

\begin{tabular}{|c|c|c|c|}
\hline \multicolumn{1}{|c|}{} & \\
\hline
\end{tabular}

\subsection{Hardness analysis}

HRC hardness test was conducted on molten sample at room temperature. A total of five HRC hardness tests were conducted and their average was taken as the representative hardness value. The average Rockwell C Hardness (HRC) value of 51.4 was measured for the VIM molten sample. This result is quite high as compared to the work of Moon et al. [11]. Therefore, heat treatment is required after melting. Some researchers have already shown that the hardness of $\mathrm{Ti}$ and its alloys increases due to increased impurities such as $\mathrm{O}, \mathrm{C}$ and $\mathrm{N}_{2} . \mathrm{N}_{2}, \mathrm{O}$, and $\mathrm{C}$ reduce impact toughness of the material and increase the brittleness (Moon et al., 2017), (Oh, \& Lim, 2016), (Su et al., 
2009), (Vutova et al., 2010), (Roh et al., 2014), (Ahmet et al., 2017), (Sazak, 1999), (Rudnev et al., 2003), (Formanoir et al., 2016), (Balla et al., 2014), ), (Suryanarayana, 2001), (Safdar et al., 2012), (Reginster et al., 2013), (Qiu, \& Attallah, 2013), (Vilaro et al., 2012), (Amato et al., 2012), ( Thijs et al., 2010), (Facchini et al., 2010), (Wasz et al., 1996). It was shown by EDS that $\mathrm{C}, \mathrm{O}$ and $\mathrm{N}_{2}$ ratios are above limits.

\section{Results and Discussions}

With the increased awareness of the circular economy, the recovery of precious materials is of great importance for the materials world and the environment. In this study, we have carried out an important study to convert the waste titanium scrap into molten titanium. It has been clearly shown that scrap titanium obtained from implant industry can be melted and cast titanium alloy has been obtained. Detailed analysis showed that we could obtain high purity titanium alloy with the scrap. We believe that the technique developed here will help the researchers to find new ways to recycle the metallic materials and this will open many fruitful papers in the scientific community and for the industrial applications. For future studies, advanced chip cleaning and purification should be carried to obtain higher quality melted products.

\section{Acknowledgements}

The authors would like to thank Metrosan Medical Company (Manisa, Turkey) for providing the raw materials and Ervak Makine Company for using VIM machine. Thanks are also Birim Makina Company for their support to the compressed into a cylindrical briquette.

\section{Author Statement}

Erdoğan Teke: Conception and design of study, Methodology, Writing- Original draft preparation. Mücahit Sütçü: Data curation, Writing- Original draft preparation. Yıldıray Başkurt: Conception and design of study, Investigation. M. Özgür Seydibeyoğlu: Supervision, Writing- Reviewing and Editing.

\section{Conflict of Interest}

The authors declare no conflict of interest.

\section{References}

Ahmed, N., Abdo, B.M., Darwish, S., Moiduddin, K., Pervaiz, S., Alahmari, A.M., \& Naveed, M. (2017). Electron beam melting of titanium alloy and surface finish improvement through rotary ultrasonic machining, The International Journal of Advanced Manufacturing Technology, 92, 3349-3361.

Amato, K.N., Gaytan, S.M., Murr, L.E., Martinez, E., Shindo, P.W., Hernandez, J., Collins, J., \& Medina, F. (2012). Microstructures and mechanical behavior of Inconel 718 fabricated by selective laser melting, Acta Materialia, 60, 2229-2239.

ASM Handbook, (2008). Casting, Volume 15, USA, ASM International.

Balla, V.K., Soderlind, J., Bose, S., \& Bandyopadhyay, A. (2014). Microstructure, mechanical and wear properties of laser surface melted Ti6Al4V alloy, Journal of 
Mechanical Behavior of Biomedical Materials, 32, 335-344.

Destefani, J.D. (1990). Introduction to Titanium and Titanium Alloys. Properties and selection: Nonferrous alloys and special-purpose materials, Volume 2, ASM International, Ohio, 586-591.

Dikici, T., \& Sutcu, M. (2017). Effects of disc milling parameters on the physical properties and microstructural characteristics of Ti6Al4V powders, Journal of Alloys and Compounds, 723, 395-400.

Donachie Jr., M.J. (2000). Titanium: A Technical Guide, ASM International, USA.

Facchini, L., Magalini, E., Robotti, P., \& Molinari, A. (2009). Microstructure and mechanical properties of Ti-6Al-4V produced by electron beam melting of prealloyed powders, Rapid Prototyping Journal, 15(3), 171-178.

Facchini, L., Magalini, E., Robotti, P., Molinari, A., Hoges, S., \& Wissenbach, K. (2010). Ductility of a Ti-6Al-4V alloy produced by selective laser melting of prealloyed powders, Rapid Prototyping Journal, 16, 450-459.

Formanoir, C., Michotte, S., Rigo, O., Germain, L., \& Godet, S. (2016). Electron beam melted Ti-6Al-4V: Microstructure, texture and mechanical behavior of the asbuilt and heat-treated material, Materials Science E Engineering A, 652, 105-119.

Froes, F.H. (2013). Powder Metallurgy of Titanium Alloys. Advances in Powder Metallurgy, Woodhead Publishing, Cambridge, 202-240.

Leyens, C. \& Peters, M. (2003). Titanium and Titanium Alloys: Fundamentals and Applications. Wiley-Vch, Weinheim.

Moon, B., Seo, J. H., Lee, H., Jung, K. H., Park, J. H., \& Jung, H. (2017). Method of recycling titanium scraps via the electromagnetic cold crucible technique coupled with calcium treatment, Journal of Alloys and Compounds, 727, 931-939.

Neff, D.V. (1990). Recycling of Nonferrous Alloys, in: ASM Handbook Committee (Eds.), Properties and selection: Nonferrous alloys and special-purpose materials, volume 2, ASM International, Ohio, 1205-1232.

Oh, J., Roh, K., \& Lim, J. (2016). Brief review of removal effect of hydrogen-plasma arc melting on refining of pure titanium and titanium alloys, International Journal of Hydrogen Energy, 41, 23033 - 23041.

Qiu, C., Adkins, N., \& Attallah, M. (2013). Microstructure and tensile properties of selectively laser-melted and of HIPed laser-melted Ti-6Al-4V, Materials Science and Engineering: A, 578(20), 230-239.

Rack, H.J., \& Qazi, J.I. (2006). Titanium alloys for biomedical application, Materials Science and Engineering: C, 26(8), 1269-1277.

Reginster, S. et al., (2013). Processing of Ti alloys by additive manufacturing: a comparison of the microstructures obtained by laser cladding, selective laser melting and electron beam melting, Materials Science Forum, 765, 413-417.

Roh, K., Suh, C., Oh, J., Kim, W., Kwon, H., \& Lim, J. (2014). Comparison of deoxidation capability for preparation of low oxygen content powder from TiNi alloy scraps, Powder Technology, 253, 266-269.

Rudnev, V., Loveless, D., Cook, R., \& Black, M. (2003). Handbook of Induction Heating, Marcell Dekker Inc., New York. 
Safdar, A. et al., (2012). Evaluation of microstructural development in electron beam melted Ti-6Al-4V, Materials Characterization, 65, 8-15.

Sazak, B.S. (1999). Design of a 500W Resonant Induction Heater, Pamukkale University Journal of Engineering Sciences, 5(1), 871-878.

Su, Y., Wang, L., Luo, L., Jiang, X., Guo, J., \& Fu, H. (2009). Deoxidation of Titanium alloy using hydrogen, International Journal of Hydrogen Energy, 34, 8958 - 8963.

Suryanarayana, C. (2001). Mechanical alloying and milling, Progress in Materials Science, $46,1-184$.

Thijs, L., Verhaeghe, L., Craeghs, T., Humbeeck, J.V., Kruth, J.P. (2010). A study of the microstructural evolution during selective laser melting of Ti-6Al-4V, Acta Materialia, 58, 3303-3312.

Topolski, S., Bochniak, W., Lagoda, M., Ostachowski, P., \& Garbacz, H. (2017). Structure and properties of titanium produced by a new method of chip recycling, Journal of Materials Processing Technology, 248, 80-91.

Vilaro, T., Colin, C., Bartout, J.D., Naze, L., \& Sennour, M. (2012). Microstructural and mechanical approaches of the selective laser melting process applied to a nickel-base superalloy, Materials Science and Engineering: A, 534, 446-451.

Vutova, K., Vassileva, V., Koleva, E., Georgieva, E., Mladenov, G., Mollov, D., \& Kardjiev, M. (2010). Investigation of electron beam melting and refining of titanium and tantalum scrap, Journal of Materials Processing Technology, 210(8), 1089-1094.

Wasz, M., Brotzen, F., McLellan, R., \& Griffin, A. (1996). Effect of oxygen and hydrogen on mechanical properties of commercial purity titanium, International Materials Reviews, 41(1), 1-12. 